\title{
Availability, perceptions, and characteristics of antibiograms among Illinois pediatricians
}

\author{
Kathryn M Spiekerman' \\ Sameer J Patel ${ }^{2,3}$ \\ Rupal Patel ${ }^{4}$ \\ Larry K Kociolek ${ }^{2,3}$
}

'School of Public Health, University of Illinois at Chicago, ${ }^{2}$ Division of Pediatric Infectious Diseases, Ann \& Robert H. Lurie Children's Hospital of Chicago, ${ }^{3}$ Department of Pediatrics, Northwestern University Feinberg School of Medicine, ${ }^{4}$ Department of Pharmacy, Ann \& Robert H. Lurie Children's Hospital of Chicago, Chicago, IL, USA
Correspondence: Larry K Kociolek Division of Pediatric Infectious Diseases, Ann \& Robert H. Lurie Children's Hospital of Chicago, 225 E. Chicago Ave, Box 20, Chicago, IL 606II, USA

Tel +l 3I 22274080

$\mathrm{Fax}+\mathrm{I} 3122279709$

Email Larry-Kociolek@northwestern.edu
This article was published in the following Dove Press journal:

Infection and Drug Resistance

5 December 2016

Number of times this article has been viewed

Abstract: Despite the enormous volume of antibiotics prescribed by pediatricians, resources to promote judicious antibiotic use are primarily limited to hospitals. The primary objective of this survey was to delineate the availability, characteristics, and perceptions of antibiograms among pediatricians. As a secondary objective, we sought to delineate the availability of other infectious diseases (ID)-related educational resources among pediatricians, the perceived need for additional resources, and their general educational preferences. We developed an anonymous electronic survey using the Research Electronic Data Capture tool, and it was sent via email to all members of the Illinois Chapter of the American Academy of Pediatrics (ICAAP). Participants were excluded if they had not completed pediatrics residency or if they had not prescribed antibiotics in the past month. Of the 1,825 ICAAP members, 294 (16\%) responded; of these, $239(81 \%)$ were eligible and completed the survey. Of the 239 respondents, $139(58 \%)$ had access to an antibiogram and 60 (25\%) had access to a pediatric-specific antibiogram. Access to a pediatric-specific antibiogram was associated with subspecialty training $(P=0.007)$ and practice location in Chicago $(P<0.0001)$. Antibiogram access was associated with perceiving being informed about resistance patterns at the national $(67 \%$ vs $54 \%, P=0.043)$ and local ( $76 \%$ vs $45 \%, P<0.0001)$ levels. Nearly all $(95 \%)$ respondents would probably or definitely use a single antibiogram that compiled antibiotic resistance data from children with common infections throughout the region. More than $75 \%$ of respondents identified both the American Academy of Pediatrics Red Book and online medical resources among the top three most useful and most frequently accessed educational resources. In addition, $91 \%$ of respondents utilized smartphones/tablets. These data suggest that there is an unmet need for additional educational resources to guide antibiotic prescribing among Illinois pediatricians. In addition, an electronic regional antibiogram would be well received and could potentially improve knowledge of antibiotic resistance and empiric antibiotic use.

Keywords: antibiotic stewardship, antibiogram, survey, pediatrics

\section{Introduction}

Judicious antibiotic use, promoted through antibiotic stewardship programs (ASPs), ${ }^{1,2}$ can slow the emergence of antibiotic resistance ${ }^{3,4}$ and reduce costs. ${ }^{5,6}$ Despite the substantial volume of antibiotics prescribed from outpatient settings, ${ }^{7,8}$ a significant proportion of which is inappropriate, ${ }^{8,9}$ inconsistent with guidelines, ${ }^{10}$ and highly variable across pediatric outpatient practices, ${ }^{11}$ ASPs are primarily limited to hospitals. While outpatient ASPs utilizing audit and feedback are an effective resource for optimizing antibiotic use, ${ }^{12}$ pediatricians may be skeptical of outpatient ASPs, ${ }^{13}$ and the benefits of this intervention may not persist once it is discontinued. ${ }^{14}$ Because of 
these challenges, a lack of physician buy-in and structural and financial barriers limit the effectiveness of outpatient stewardship. Because there is an unmet need for more cost-effective antibiotic stewardship-related resources for pediatricians, the primary objective of this survey was to delineate the availability, characteristics, and perceptions of antibiograms among pediatricians. As secondary objective, we sought to delineate the availability of other infectious diseases (ID)-related educational resources among pediatricians, the perceived need for additional resources, and their general educational preferences.

\section{Materials and methods}

We conducted an anonymous electronic survey using the Research Electronic Data Capture (REDCap) ${ }^{15}$ tool at Northwestern University over a 4-week period in October 2015. The survey was sent out via email to all 1,825 members of the Illinois Chapter of the American Academy of Pediatrics (ICAAP). Each participant was assigned a unique survey link to prevent multiple surveys being submitted by an individual participant. Captured survey data included geographic location, residency and subspecialty training, practice characteristics, antibiotic prescribing frequency, availability and perceptions of various ID-related resources, and preferences for educational resources. Participants were excluded if they had not completed residency training in pediatrics or if they had not prescribed antibiotics in the past month. For each completed survey, a donation was made to one of three pediatric-related charities chosen by the participants. Survey responses were coded by and stored anonymously in REDCap. For Likert scaled responses, data were dichotomized. A cross-sectional study was performed to identify characteristics associated with resource access, perceptions of resource value, and educational preferences. Proportions were compared using chi-square tests. Prevalence ratios and 95\% confidence intervals were calculated using binomial regression analysis. Variables with $P$-values $<0.1$ were included in the multivariate regression model. Twosided $P$-value of 0.05 was considered statistically significant. Descriptive and multivariable statistics were performed using SAS version 9.4 (SAS Institute Inc, Cary, NC, USA). The Lurie Children's Institutional Review Board provided an exemption for this study and granted a waiver of informed consent.

\section{Results}

Of the 1,825 ICAAP members, 294 (16\%) responded; of these, 239 (81\%) were eligible and completed the survey. Reasons for exclusion were: no antibiotics prescribed in the past month $(\mathrm{n}=25)$; has not completed pediatric residency training $(n=29)$; and failure to complete the survey $(n=1)$. The eligible respondents included a diverse group of general and subspecialty-trained pediatricians (Table 1). The mean number of years since completing residency was 16.3 (range 1-50 years, standard deviation 11.6). The numbers of respondents practicing in the outpatient, inpatient, emergency department (ED), and urgent care settings, respectively, were $181(75.7 \%), 144(60.3 \%), 24(10.0 \%)$, and $28(11.7 \%)$.

Table I Physician demographics and associations with antibiogram access $(n=239)$

\begin{tabular}{|c|c|c|c|c|c|c|}
\hline Characteristics & Category & $\mathbf{N}(\%)$ & $\begin{array}{l}\text { Access to } \\
\text { antibiogram } \\
(\%) \\
\end{array}$ & $\begin{array}{l}\text { Prevalence ratio } \\
\text { (95\% CI, P-value) }\end{array}$ & $\begin{array}{l}\text { Access to } \\
\text { pediatric-specific } \\
\text { antibiogram (\%) }\end{array}$ & $\begin{array}{l}\text { Prevalence ratio }(95 \% \mathrm{Cl} \text {, } \\
\text { P-value) }\end{array}$ \\
\hline \multirow[t]{2}{*}{ Practice location } & Chicago & $110(46.0)$ & $73(66.4)$ & $1.30(1.05-1.61,0.018)$ & $51(69.9)$ & $5.12(3.17-8.29, \leq 0.0001)^{*}$ \\
\hline & Outside Chicago & $129(54.0)$ & $66(51.2)$ & & $9(13.6)$ & \\
\hline \multirow[t]{2}{*}{ Training } & Subspecialty & $64(26.8)$ & $52(81.3)$ & $1.63(1.31-2.04, \leq 0.0001)^{*}$ & $34(65.4)$ & $2.19(1.50-3.19, \leq 0.0001)^{*}$ \\
\hline & General pediatrics & I 75 (73.2) & $87(49.7)$ & & $26(29.9)$ & \\
\hline \multirow[t]{2}{*}{ Practice setting } & $\begin{array}{l}\text { Includes inpatient } \\
\text { and/or emergency } \\
\text { department }\end{array}$ & $159(66.5)$ & $108(67.9)$ & $1.75(1.36-2.26, \leq 0.0001)^{*}$ & $53(49.1)$ & $2.17(1.21-3.89,0.0087)$ \\
\hline & Outpatient only & $80(33.5)$ & $31(38.8)$ & & $7(22.6)$ & \\
\hline Children's hospital & Yes & $190(79.5)$ & $118(62.1)$ & $1.45(1.07-1.95,0.015)$ & $57(48.3)$ & $3.38(1.48-7.73,0.0037)$ \\
\hline privileges & No & $49(20.5)$ & $21(42.9)$ & & $3(14.3)$ & \\
\hline Academic & Yes & $155(64.9)$ & I0I (65.2) & $1.44(1.13-1.83,0.0029)$ & $50(49.5)$ & $1.88(1.14-3.12,0.0139)$ \\
\hline appointment & No & $84(35.1)$ & $38(45.2)$ & & $10(26.3)$ & \\
\hline Monthly antibiotic & $\geq 20$ & $106(44.4)$ & $67(63.2)$ & $1.16(0.94-1.45,0.16)$ & $26(38.8)$ & $0.82(0.56-1.21,0.32)$ \\
\hline prescriptions & $<20$ & I $33(55.6)$ & $72(54.1)$ & & $34(47.2)$ & \\
\hline
\end{tabular}

Notes: Prevalence ratios and $P$-values in bold indicate statistical significance $(P<0.05)$ on bivariate analysis. *Statistically significant prevalence ratios on multivariate analysis. Abbreviation: $\mathrm{Cl}$, confidence interval. 
Overall, 225 (94\%) pediatricians had access to a pediatric ID consultant; 10/14 (71\%) of those without a pediatric ID specialist had access to an adult ID consultant. Consultation with an ID physician occurred at least once monthly and at least once every 3 months among 50/235 (21\%) and 102/235 (44\%) respondents, respectively. Of the 239 respondents, 139 (58\%) had access to an antibiogram and $60(25 \%)$ had access to a pediatric-specific antibiogram; $22(16 \%)$ and $60(43 \%)$ review their antibiogram at least once a month and once every 3 months, respectively. On bivariate analyses, practice location in Chicago, practice setting that includes inpatient and/or the ED, subspecialty training, privileges at a children's hospital, and having a medical school academic appointment, but not antibiotic prescription volume, were significantly $(P<0.05)$ associated with both having access to an antibiogram and a pediatricspecific antibiogram (Table 1). On multivariate analysis, access to an antibiogram was associated with subspecialty training $(P=0.006)$ and practice setting that includes inpatient and/or the $\operatorname{ED}(P=0.003)$; access to a pediatric-specific antibiogram was associated with subspecialty training $(P=0.007)$ and practice location in Chicago $(P<0.0001)$. Of the 239 respondents, 147 (62\%) reported being a great deal or moderately informed about national antibiotic resistance patterns for pathogens causing common pediatric infections (ie, acute otitis media, community-acquired pneumonia, pharyngitis, urinary tract infection, and skin and soft tissue infection) and 150 (63\%) reported being a great deal or moderately informed about local antibiotic resistance patterns. Access to an antibiogram was associated with being informed about antibiotic resistance both at the national ( $67 \%$ vs $54 \%, P=0.043)$ and local ( $76 \%$ vs $45 \%, P<0.0001)$ levels.

Antibiograms were electronically accessible by $109 / 139$ (78\%) respondents and were issued by a hospital to nearly all of the respondents (137/139 [99\%]); the remaining two respondents had an antibiogram issued by a nonhospital entity. Among the 139 pediatricians with access to an antibiogram, $121(87 \%)$ agreed or strongly agreed that it is useful for understanding trends in antibiotic resistance, and $115(83 \%)$ agreed or strongly agreed that it is useful for guiding empiric antibiotic selection. Sixty-five (47\%) respondents reported the need for additional education for antibiogram interpretation and guiding empiric antibiotic selection. The following antibiogram limitations were identified by respondents: mostly infections from hospitalized and/or medically complex patients (58\%), mostly adult patients $(38 \%)$, too few pediatric patients $(24 \%)$,
Table 2 Pediatrician educational resource preferences

\begin{tabular}{lll}
\hline Resource & $\begin{array}{l}\text { Top } 3 \\
\text { most useful } \\
\text { resources (\%) }\end{array}$ & $\begin{array}{l}\text { Top } 3 \text { most } \\
\text { frequently accessed } \\
\text { resources (\%) }\end{array}$ \\
\hline AAP Red Book & 83.3 & 89.1 \\
Online resources & 79.9 & 78.2 \\
Peer-reviewed journals & 47.3 & 48.1 \\
Live lectures/conferences & 29.3 & 23.8 \\
Textbooks & 25.9 & 23.8 \\
Smartphone apps & 20.5 & 25.1 \\
Webinars & 3.3 & 1.7 \\
\hline
\end{tabular}

Abbreviation: AAP, American Academy of Pediatrics.

and poorly representative of patients from their practice's geographical location (5\%). Among the 100 pediatricians without access to an antibiogram, $88(88 \%)$ agreed that an antibiogram would improve their understanding of trends in antibiotic resistance and $83(83 \%)$ agreed that it would improve empiric antibiotic selection. A vast majority of respondents (98\%) would probably or definitely use additional educational resources describing local antibiotic resistance; $95 \%$ of respondents would probably or definitely use a single antibiogram that compiled antibiotic resistance data from children with common pediatric infections throughout the region.

Among the 239 respondents, 213 (89\%) indicated their educational preferences by identifying their top three most useful and most frequently accessed resources (Table 2). More than $75 \%$ of respondents identified both the American Academy of Pediatrics (AAP) Red Book and online medical resources among the top three most useful and most frequently accessed educational resources. Despite smartphones/tablets being reportedly used by $91 \%$ and $52 \%$ of respondents for personal and professional use, respectively, the electronic version of the AAP Red Book was used by only $34 \%$ of respondents. While fewer years since completing residency was associated with the use of online medical resources (14.2 vs 22.6 years, $P<0.0001$ ), there was no difference in time since residency among those using smartphones/tablets ( 16.3 vs $16.5, P=0.90$ ) or the online version of the AAP Red Book (15.7 vs 16.6, $P=0.58$ ).

\section{Discussion}

These data suggest that there is an unmet need among Illinois pediatricians for additional resources and education related to antibiotic resistance and antibiotic prescribing. Nearly $40 \%$ of respondents do not feel well informed about either national or local antibiotic resistance rates for pathogens causing common pediatric infections (ie, acute otitis media, community-acquired pneumonia, pharyngitis, 
urinary tract infection, and skin and soft tissue infection). Although those with access to an antibiogram reported being better informed about antibiotic resistance rates, only half of the respondents have access to an antibiogram, and only one-quarter of respondents have access to a pediatricspecific antibiogram.

In a multicenter cluster randomized trial, Gerber et al demonstrated the improvement in antibiotic prescribing patterns among sites with ASPs utilizing provider education and quarterly audit and feedback of antibiotic prescribing data. ${ }^{12}$ However, 18 months after the ASPs had been discontinued following the completion of this study, the benefits of the ASPs on antibiotic prescribing patterns were lost. ${ }^{14}$ These studies highlighted the importance of ongoing provider education. Because outpatient ASPs may not be feasible, well accepted, and/or cost-effective for many pediatric practices, these data highlight the importance of equipping pediatricians with the tools and education needed to incorporate stewardship into their daily prescribing.

Lack of access to an antibiogram is particularly common among general pediatricians, those practicing outside of the Chicago area, and those physicians who work exclusively in the outpatient setting (ie, common characteristics among community pediatricians). Furthermore, frequency of antibiotic prescribing did not vary among those with and without access to an antibiogram. Because of the enormous volume of antibiotics prescribed in the outpatient setting, ${ }^{7,8}$ provision of antibiograms to community pediatricians may be an important antibiotic stewardship strategy, particularly because outpatient antibiotic prescribing is highly variable and/or oftentimes inappropriate..$^{8-11}$ This unmet need is additionally highlighted because pediatricians both with and without access to an antibiogram generally viewed it as an important resource for understanding trends in antibiotic resistance and guiding empiric antibiotic selection. Despite the favorable view and perceived benefits of an antibiogram, many limitations of an antibiogram were identified, particularly the poor representation of relatively healthy children outside of the hospital setting.

Professional society guidelines suggest that antibiotic resistance patterns in the prescriber's geographic region should impact prescribing behaviors. For example, guidelines for dosing of empiric therapy for suspected pneumococcal pneumonia in children are based on the local rate of penicillin resistance, ${ }^{16}$ and guidelines for empiric therapy for suspected methicillin-resistant Staphylococcus aureus infections are based on the local rate of clindamycin resistance. ${ }^{17}$ In addition, rates of multidrug resistance among Escherichia coli isolates in the pediatric ambulatory setting are increasing, ${ }^{18}$ and there has been a shift in the antibiogram of $S$. aureus (declining resistance to anti-staphylococcal beta-lactam antibiotics and increasing resistance to clindamycin) in children. ${ }^{19}$ These notable changes in the epidemiology of common pediatric pathogens reinforce the importance of keeping pediatricians apprised of antibiotic resistance patterns in their communities to guide appropriate antibiotic prescribing. This stewardship goal could potentially be achieved by compiling antibiotic resistance data throughout the region and developing a regularly updated and electronically accessible antibiogram of pathogens isolated from children with common pediatric infections. This would require the close collaboration of academic and community hospitals serving that particular region. Leveraging existing biomedical informatics tools would assist in this process. Pediatricians in this study responded enthusiastically to the potential for such a resource.

In this study, pediatricians identified the AAP Red Book and online medical resources among the most useful and most frequently accessed resources for antibiotic prescribing for their pediatric patients. Furthermore, the vast majority of respondents reported using a smartphone/tablet. Thus, we envision an electronic resource that presents compiled antibiotic resistance data from pediatric pathogens throughout the region and could be accessed by pediatricians via smartphones/tablets in the office or hospital. For example, because knowledge of pneumococcal, staphylococcal, and $E$. coli resistance rates in the community impact empiric antibiotic selection, the existing AAP Red Book smartphone/ tablet application could be enhanced by providing links to this electronic antibiogram that is specific to the users' specified geographical location. It is conceivable that the capabilities of such an electronic resource could be expanded to include additional ID-related educational materials, such as vaccine updates from the Advisory Committee on Immunization Practices, local ID outbreak notifications from the local health departments, antibiotic manufacturing shortages, etc.

\section{Limitations}

This study is limited by the relatively low response rate. Bias may be introduced because respondents may have had a particular interest in the survey topic. However, the response rate was similar to other ICAAP surveys, and the number of respondents was high. Furthermore, the survey was limited to Illinois pediatricians and members of ICAAP. Thus, the data presented may not be generalizable. For example, it would be expected that compared with nonmembers, ICAAP members 
would be more likely to access the AAP Red Book. Although we do not know the characteristics of nonrespondents, our data suggest that we successfully captured responses from a diverse group of community and academic general and subspecialty pediatricians.

\section{Conclusion}

Because formal ASPs are lacking in the vast majority of outpatient settings, equipping pediatricians with the necessary tools for understanding antibiotic resistance is an important step in promoting the incorporation of stewardship into physician practices. These data suggest that many Illinois pediatricians perceive themselves as relatively uninformed about antibiotic resistance, many lack access to an antibiogram, and that an antibiogram is viewed favorably as an important tool for understanding antibiotic resistance and improving empiric antibiotic selection. Because the vast majority of respondents use and prefer electronic resources, this study suggests that an electronic regional antibiogram would be well received by pediatricians.

\section{Acknowledgments}

Research reported in this publication was supported, in part, by the National Institutes of Health's National Center for Advancing Translational Sciences (grant number UL1TR001422). The content is solely the responsibility of the authors and does not necessarily represent the official views of the National Institutes of Health.

\section{Disclosure}

LKK reports receiving research grant support from Merck, receiving research support (supplies) from Alere/Techlab, and being a scientific advisor for Actelion, all outside the submitted work. SJP reports receiving research grant support from Merck, outside the submitted work. The other authors report no conflicts of interest in this work.

\section{References}

1. Barlam TF, Cosgrove SE, Abbo LM, et al. Implementing an antibiotic stewardship program: guidelines by the infectious diseases society of America and the society for healthcare epidemiology of America. Clin Infect Dis. 2016;62(10):e51-e77.

2. Golding GR, Quinn B, Bergstrom K, et al. Community-based educational intervention to limit the dissemination of community-associated methicillin-resistant Staphylococcus aureus in Northern Saskatchewan, Canada. BMC Public Health. 2012;12:15.
3. Elligsen M, Walker SA, Pinto R, et al. Audit and feedback to reduce broad-spectrum antibiotic use among intensive care unit patients: a controlled interrupted time series analysis. Infect Control Hosp Epidemiol. 2012;33(4):354-361.

4. DiazGranados CA. Prospective audit for antimicrobial stewardship in intensive care: impact on resistance and clinical outcomes. Am J Infect Control. 2012;40(6):526-529.

5. Sick AC, Lehmann CU, Tamma PD, Lee CK, Agwu AL. Sustained savings from a longitudinal cost analysis of an internet-based preapproval antimicrobial stewardship program. Infect Control Hosp Epidemiol. 2013;34(6):573-580.

6. Standiford HC, Chan S, Tripoli M, Weekes E, Forrest GN. Antimicrobial stewardship at a large tertiary care academic medical center: cost analysis before, during, and after a 7-year program. Infect Control Hosp Epidemiol. 2012;33(4):338-345.

7. Chai G, Governale L, McMahon AW, Trinidad JP, Staffa J, Murphy D. Trends of outpatient prescription drug utilization in US children, 2002-2010. Pediatrics. 2012;130(1):23-31.

8. Hersh AL, Shapiro DJ, Pavia AT, Shah SS. Antibiotic prescribing in ambulatory pediatrics in the United States. Pediatrics. 2011;128(6):1053-1061.

9. Fleming-Dutra KE, Hersh AL, Shapiro DJ, et al. Prevalence of inappropriate antibiotic prescriptions among US ambulatory care visits, 2010-2011. JAMA. 2016;315(17):1864-1873.

10. Saleh EA, Schroeder DR, Hanson AC, Banerjee R. Guideline-concordant antibiotic prescribing for pediatric outpatients with otitis media, community-acquired pneumonia, and skin and soft tissue infections in a large multispecialty healthcare system. Clin Res Infect Dis. 2015;2(1):pii 110.

11. Gerber JS, Prasad PA, Russell Localio A, et al. Variation in antibiotic prescribing across a pediatric primary care network. J Pediatric Infect Dis Soc. 2015;4(4):297-304.

12. Gerber JS, Prasad PA, Fiks AG, et al. Effect of an outpatient antimicrobial stewardship intervention on broad-spectrum antibiotic prescribing by primary care pediatricians: a randomized trial. JAMA. 2013;309(22):2345-2352.

13. Szymczak JE, Feemster KA, Zaoutis TE, Gerber JS. Pediatrician perceptions of an outpatient antimicrobial stewardship intervention. Infect Control Hosp Epidemiol. 2014;35(Suppl 3):S69-S78.

14. Gerber JS, Prasad PA, Fiks AG, Localio AR, Bell LM, Keren R, Zaoutis TE. Durability of benefits of an outpatient antimicrobial stewardship intervention after discontinuation of audit and feedback. JAMA. 2014;312(23):2569-2570.

15. Harris PA, Taylor R, Thielke R, Payne J, Gonzalez N, Conde JG. Research electronic data capture (REDCap)--a metadata-driven methodology and workflow process for providing translational research informatics support. J Biomed Inform. 2009;42(2):377-381.

16. Bradley JS, Byington CL, Shah SS, et al. Executive summary: the management of community-acquired pneumonia in infants and children older than 3 months of age: clinical practice guidelines by the Pediatric Infectious Diseases Society and the Infectious Diseases Society of America. Clin Infect Dis. 2011;53(7):617-630.

17. Liu C, Bayer A, Cosgrove SE, et al. Clinical practice guidelines by the Infectious Diseases Society of America for the treatment of methicillinresistant Staphylococcus aureus infections in adults and children. Clin Infect Dis. 2011;52(3):e18-e55.

18. Logan LK, Braykov NP, Weinstein RA, Laxminarayan R. Extendedspectrum $\beta$-lactamase-producing and third-generation cephalosporinresistant enterobacteriaceae in children: trends in the United States, 1999-2011. J Pediatric Infect Dis Soc. 2014;3(4):320-328.

19. Sutter DE, Milburn E, Chukwuma U, Dzialowy N, Maranich AM, Hospenthal DR. Changing susceptibility of staphylococcus aureus in a US pediatric population. Pediatrics. 2016;137(4):pii:e20153099. 


\section{Publish your work in this journal}

Infection and Drug Resistance is an international, peer-reviewed openaccess journal that focuses on the optimal treatment of infection (bacterial, fungal and viral) and the development and institution of preventive strategies to minimize the development and spread of resistance. The journal is specifically concerned with the epidemiology of antibiotic resistance and the mechanisms of resistance development and diffusion in both hospitals and the community. The manuscript management system is completely online and includes a very quick and fair peerreview system, which is all easy to use. Visit http://www.dovepress.com/ testimonials.php to read real quotes from published authors.

Submit your manuscript here: https://www.dovepress.com/infection-and-drug-resistance-journal 